\title{
Efficacia del rituximab in pazienti con oftalmopatia basedowiana di grado medio-grave attiva: uno studio randomizzato controllato
}

\author{
Michele Marinò ${ }^{1}$
}

Pubblicato online: 15 aprile 2015

(C) Springer International Publishing AG 2015

\section{Commento a:}

Efficacy of B-cell targeted therapy with rituximab in patients with active moderate to severe Graves' orbitopathy: a randomized controlled study. M. Salvi, G. Vannucchi, N. Currò, I. Campi, D. Covelli, D. Dazzi, S. Simonetta, C. Guastella, L. Pignataro, S. Avignone, P. Beck-Peccoz.

J Clin Endocrinol Metab (2015) 100(2):422-431

Nel precedente numero abbiamo commentato i risultati, sostanzialmente negativi, di uno studio americano randomizzato rituximab vs placebo in pazienti con oftalmopatia basedowiana $(\mathrm{OB})$ di grado moderato-grave, attiva [1]. In questo numero commentiamo i risultati di uno studio italiano analogo, nel quale il rituximab veniva confrontato con i glucocorticoidi, con risultati del tutto opposti rispetto al precedente studio americano.

Si tratta di uno studio in doppio cieco, randomizzato, che comprendeva 32 pazienti con oftalmopatia di grado mediograve, attiva, suddivisi in due gruppi di trattamento: glucocorticoidi endovena (7,5 g totali di metilprednisolone) e rituximab (2000 o $500 \mathrm{mg}$ ). Gli end point principali dello studio erano la riduzione del clinical activity score di almeno 2 punti o una riduzione dello stesso al di sotto dei 3 punti dopo 24 settimane. Gli end point secondari erano le variazioni di esoftalmo, apertura palpebrale, diplopia, motilità oculare e qualità della vita.
Benché venisse osservata una significativa riduzione del clinical activity score in entrambi i gruppi, la riduzione era significativamente più marcata nei pazienti trattati con rituximab, con una risposta favorevole nel $100 \%$ dei casi a 24 settimane. In nessun paziente trattato con rituximab veniva osservata una riattivazione della malattia, che veniva invece osservata dopo terapia con glucocorticoidi endovena.

Come menzionato sopra, questi risultati sono in forte contrasto con quelli dello studio americano sullo stesso argomento, e sono invece in linea con quelli di studi precedenti non randomizzati. Le possibili spiegazioni di questa discrepanza sono l'esiguo numero di pazienti in entrambi gli studi e la possibile diversa selezione degli stessi. A fronte di dati sicuramente incoraggianti, permangono dei dubbi in merito alla possibilità di utilizzare il rituximab nella pratica clinica, anche alla luce dei suoi effetti collaterali. Per poter chiarire definitivamente se questa terapia può essere o meno efficace, sono senz'altro necessari studi più ampi che dimostrino un miglior rapporto benefici/effetti collaterali rispetto ai glucocorticoidi, che rimangono al momento la terapia medica di prima scelta per l'oftalmopatia basedowiana.

\section{Bibliografia}

1. Stan MN, Garrity JA, Carranza Leon BG et al (2015) Randomized controlled trial of rituximab in patients with Graves' orbitopathy. J Clin Endocrinol Metab 100(2):432-441
M. Marinò

michele.marino@md.unipi.it

1 U.O. Endocrinologia 1, Università di Pisa, Pisa, Italia 